\title{
Simple experiment on the sputtering rate of solids in gas discharges
}

\author{
Peter Hartmann, ${ }^{1,2, a)}$ Jorge C. Reyes, ${ }^{2}$ Ihor Korolov, ${ }^{1}$ Lorin S. Matthews, ${ }^{2}$ \\ and Truell W. Hyde ${ }^{2}$ \\ ${ }^{1}$ Institute for Solid State Physics and Optics, Wigner Research Centre for Physics, Hungarian Academy \\ of Sciences, P.O. Box 49, H-1525 Budapest, Hungary \\ ${ }^{2}$ Center for Astrophysics, Space Physics and Engineering Research (CASPER), One Bear Place 97310, \\ Baylor University, Waco, Texas 76798, USA
}

(Received 13 February 2017; accepted 15 May 2017; published online 1 June 2017)

\begin{abstract}
We present a very simple and sensitive method to measure the sputtering rate of solid materials in stationary low-pressure gas discharges. The method is based on the balance of the centrifugal force and the confinement electric force acting on a single electrically charged dust particle in a rotating environment. We demonstrate the use and sensitivity of this method in a capacitively coupled radio frequency argon discharge. We were able to detect a reduction of $10 \mathrm{~nm}$ in the diameter of a single dust particle. Published by AIP Publishing. [http://dx.doi.org/10.1063/1.4985080]
\end{abstract}

Low-pressure gas discharges in industrial and laboratory applications are mostly confined in a vacuum chamber made of metallic and/or dielectric solid materials. These surfaces define the boundary conditions (temperature, electric potential, etc.) and provide the most significant loss (and sometimes source) channels for the charged particles by electric conduction, surface recombination, electron emission, etc. As gas discharges are driven-dissipative systems, where the charge production processes (ionization and emission) and loss channels (recombination and absorption) are separated in both space and time, the geometrical properties and the boundary conditions have significant effects on the overall plasma parameters such as the distributions of charged species densities, temperature, and electric potential. To date the effect of solid surfaces at the edges of gas discharge plasmas can only be taken into account by applying overly simplified phenomenological models. ${ }^{1}$ The data for the different processes in these models, like secondary electron emission yield, electron reflection probability, sputtering yield, etc., are not available in general for any combination of gas and solid materials. In the rare cases where there are experimental data published, the experiments are mostly performed in a high vacuum environment using mono-energetic beams of electrons or ions and clean surfaces. ${ }^{2,3}$ These conditions are very different from discharge conditions, where the background gas is able to modify the surface.

To advance the field of gas discharge physics, a deeper understanding of the mutual interaction between the discharge plasma and the surfaces is needed. Increasingly, effort is being put into research targeting the combined, self-consistent description of the solid-plasma system, the so called "plasma interface" at the microscopic level of individual atoms and electrons including quantum effects. $^{4}$ At some stage of these efforts, any new results will have to be validated against actual phenomenological models and experimental data obtained under real discharge conditions.

\footnotetext{
${ }^{\text {a)} E l e c t r o n i c ~ m a i l: ~ h a r t m a n n . p e t e r @ w i g n e r . m t a . h u ~}$
}

The aim of this study is to provide a simple and fundamental experimental technique for determination of the sputtering rate of solid materials in gas discharges with high accuracy and sensitivity. ${ }^{5}$ This method can be used to provide experimental data useful for both present phenomenological models and future fundamental models describing sputtering, one of the important processes occurring during the interaction of plasmas with solid surfaces. To achieve this we combine the high sensitivity of dusty plasmas to the force balance in gas discharges ${ }^{6}$ with the capability of finetuning of inertial forces in a fully controlled rotating environment to high accuracy. Similar technique was used already by Carstensen et al. ${ }^{7}$ to measure dust particle charge and Debye screening length.

In addition to the fundamental studies of the plasmasurface interaction, our new method also provides information on the reduction of the size of dust particles, data which are directly applicable in the field of strongly coupled dusty plasma research, ${ }^{8}$ where in practically all studies it is assumed that the size (and with this the charge and mass) of the dust grains remains constant over the time span of the experiments. However, from time to time attempts have been made by the dusty plasma community to investigate the size reduction of dust particles in gas discharges by applying different variants of the Mie-scattering method. ${ }^{9,10}$ Stoffels et al. ${ }^{11}$ studied the reactive etching in oxygen containing plasma, while Killer et al. applied ellipsometry and tomography methods. ${ }^{12,13}$ Alternatively the variations of particle oscillation frequencies were also used to estimate particle size changes. ${ }^{14,15}$ Experiments in quadrupole traps ${ }^{16}$ even revealed that mass loss can occur without the presence of a discharge, through the outgassing of water vapor from the particles.

Our new experimental technique consists of two stages. First, after stabilizing the discharge conditions (gas pressure and driving power) the horizontal confining electric field is measured by changing the rotation rate. Second, the variation of the charge to mass ratio $(Q / m)$ of the dust grain is measured over a long time period at constant rotation rate. In more detail: 
Stage 1: The dust particle is negatively charged and is levitated in the sheath electric field above the lower, horizontal, powered electrode. The dust particle is confined within a glass cylinder placed on the lower electrode. The walls of the cylinder develop a negative floating potential, providing a horizontal electric field. The powered electrode is able to rotate, and the rotating environment drags the gas inside the glass cylinder. Within a short period of time (about a second), the rotation inside the cylinder becomes uniform, like that of a rigid body with every object revolving on circular orbits around the rotation axis. The force balance of the levitating dust particle is now modified: a centripetal acceleration is needed to sustain the circular orbit. For a given rotation rate the dust particle orbital radius is defined by the centripetal acceleration, which is equal to the acceleration due to the horizontal electrostatic confinement

$$
a_{\mathrm{cp}}=r \omega^{2}=\frac{Q}{m} E(r),
$$

where $r$ is the radial position measured from the center of rotation, $\omega$ is the angular velocity, and $Q$ and $m$ are the charge and mass of the dust grain. At this stage, the orbital radius is measured for a set of angular velocities resulting in the relation $r(\omega)$, which being a monotonic function ${ }^{6}$ is inverted into the more useful function $\omega_{0}(r)$. Using this measured relationship, the horizontal electric field distribution is easily derived from Eq. (1)

$$
E(r)=\frac{m}{Q} r \omega_{0}^{2}(r) .
$$

Here, we emphasize that $\mathrm{E}(\mathrm{r})$ is independent of the dust particle properties, since it is defined purely by the gas discharge and its boundary conditions.

Stage 2: After mapping $E(r)$ in stage 1, the very same dust particle in the same discharge is observed for a long time (approximately $1 \mathrm{~h}$ ) at a constant angular velocity $\Omega$. The measured quantity is the orbital radius vs. time $r(t)$. The horizontal force balance is still described by Eq. (1), which can be rearranged into

$$
\frac{m}{Q}(t)=\frac{E(r(t))}{\Omega^{2} r(t)}=\frac{m_{0}}{Q_{0}} \frac{\omega_{0}^{2}(r(t))}{\Omega^{2}},
$$

where the " 0 " index refers to the initial quantities obtained from stage 1 . The common theories of dust particle charging $^{17}$ assume a linear relationship between the electric charge and the dust particle radius $(R)$, as in the case of the capacitance of an ideal spherical capacitor. For this approximation to be valid two conditions should be fulfilled: (i) the Debye screening length should be larger than the grain size and (ii) the ion-neutral collision mean free path should be larger than the Debye screening length. Both conditions have to be evaluated at the position of the dust grain: bulk plasma parameters can be misleading, as the dust particles levitate in the sheath. These requirements define upper limits for grain size and gas pressure, but the following derivation can be generalized if a proper charge-size relation is available. The mass, on the other hand, scales trivially with
$R^{3}$; thus, the relative variation of the dust particle radius can be expressed from Eq. (3) as

$$
\frac{R(t)}{R_{0}}=\sqrt{\frac{Q_{0}}{m_{0}} \frac{m}{Q}(t)}=\frac{\omega_{0}(r(t))}{\Omega},
$$

where the function $\omega_{0}(r)$ is known from stage 1. We emphasize that this relative measurement method relies only on the accuracy of the angular velocity measurement and the long-time stability of the gas discharge plasma. The usual uncertainties that govern the measurement of dust parameters (charge and mass) and distance calibrations do not appear here.

The most fundamental underlying assumption of this method is that the many-fold forces acting on the dust grain (ion drag force, polarization force, ${ }^{18}$ gravity, electrode sheath electric force, etc.) contribute to the equilibrium vertical position of the grain and have only negligible contribution to the horizontal force balance.

To demonstrate the potential of this method, we have performed an experiment in a GEC reference cell at the Hypervelocity Impacts and Dusty Plasma Lab (HIDPL) of the Center for Astrophysics, Space Physics, and Engineering Research (CASPER) at Baylor University. The standard $10 \mathrm{~cm}$ (4 in.) horizontal lower powered electrode was modified with the addition of a rotatable extension as shown in Fig. 1, realizing the "RotoDust" setup. ${ }^{6}$ The DC motor (type BMU260C-A-3) is placed inside the vacuum chamber and is mounted using insulating (Teflon) parts and shaft, which connect to the rotatable electrode disk with an o-ring belt drive. The motor controller guarantees a constant rotation rate by means of active feedback and regulation. The cylindrical cutout in the center of the rotating electrode is used to hold glass cylinders of $2.5 \mathrm{~cm}$ height and different diameters.

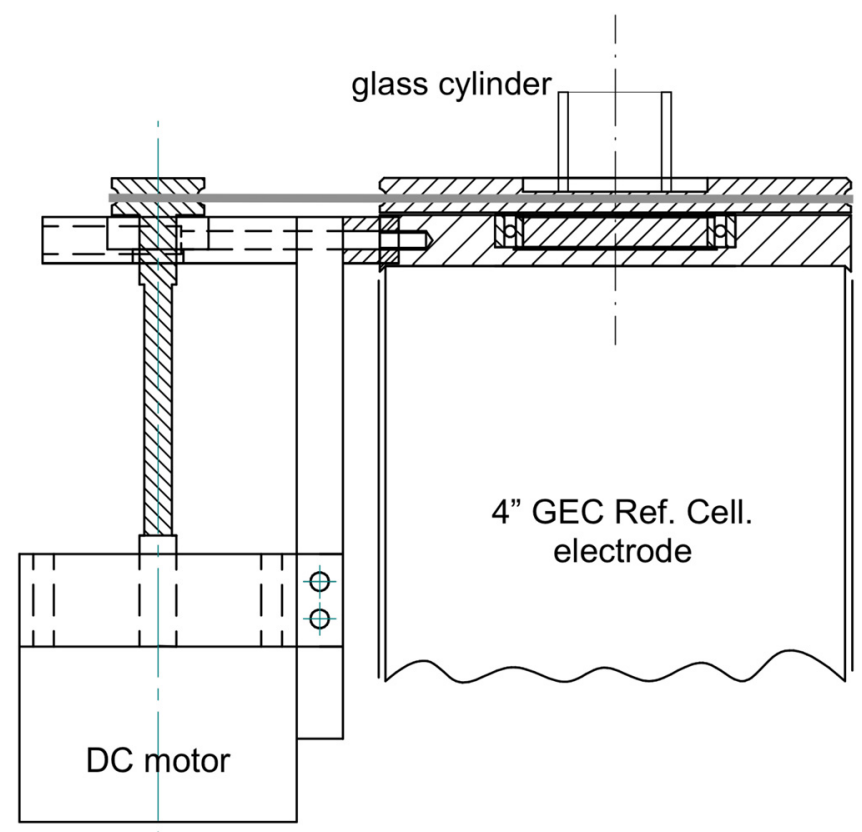

FIG. 1. Schematic drawing of the RotoDust extension installed in the GEC reference cell at the HIDPL. 


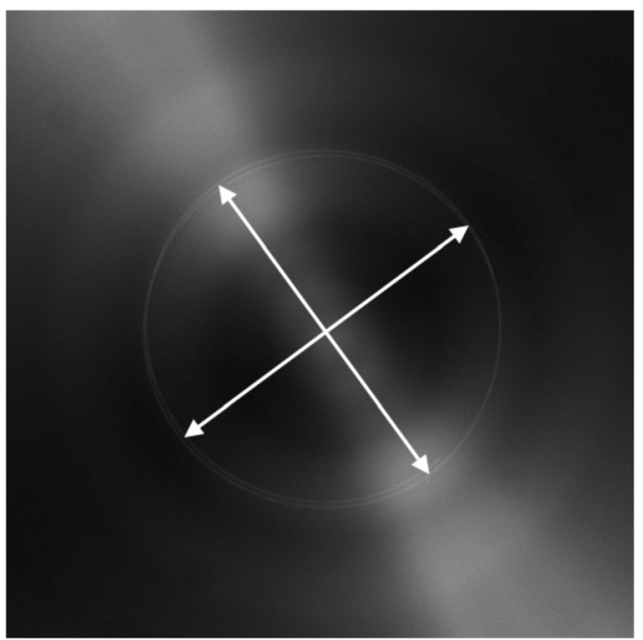

FIG. 2. Example image of a particle orbit at $\omega=26.6 \mathrm{rad} / \mathrm{s}$ angular velocity. The arrows indicate two perpendicular orbital diameter measurements, which are averaged.

Once the discharge is ignited the dust is introduced into the glass cylinder by a shaker mounted above the electrode. The following results are presented for one particular measurement, which was carried out in $14.6 \mathrm{~Pa}$ (110 mTorr) argon gas at a steady gas flow of $20 \mathrm{sccm}$. The $13.56 \mathrm{MHz}$ radio frequency $(R F)$ power was $4 \mathrm{~W}$, the electrode gap between the lower powered plane RotoDust electrode and the upper grounded ring electrode was $2.5 \mathrm{~cm}$. The glass cylinder in the center of the RotoDust electrode had an inner diameter of $10 \mathrm{~mm}$. A single melamine-formaldehyde (MF) dust particle with nominal diameter of $8.89 \mu \mathrm{m}$ was illuminated by a $532 \mathrm{~nm}$ laser sheath at $1 \mathrm{~W}$ total power. In the HIDPL, the dust particles are stored between measurements (for several months) inside the evacuated vacuum chamber assuring that the particles reach stable conditions in the low pressure environment; no outgassing needs to be assumed. Long exposure images were taken with an Allied Vision Prosilica GX1050 CCD camera such that full orbits of the revolving dust particle were captured on each image (an example is shown in Fig. 2). The diameter of the circular orbits was measured in two perpendicular directions to

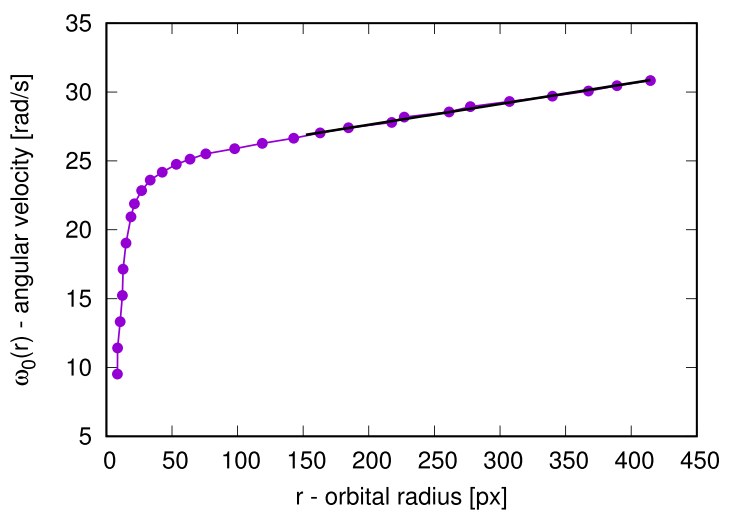

FIG. 3. Angular velocity vs. orbital radius, an intermediate result of stage 1. The distance unit is image pixels (px), as proper distance calibration is not needed for the data evaluation. The thick black line is the linear fit to data points with $r \geq 150 \mathrm{px}$.

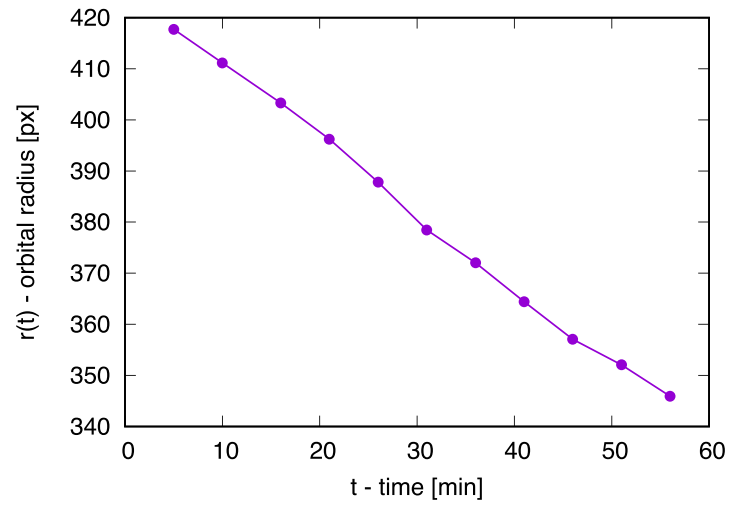

FIG. 4. Time dependent orbital radius (in units of image pixels) at $\Omega=31.2 \mathrm{rad} / \mathrm{s}$ angular velocity.

obtain the radius in units of image pixels. The $\omega_{0}(r)$ relationship is plotted in Fig. 3 .

These measurements of stage 1 were performed within a few minutes; over the next sixty minutes the angular velocity was held constant, $\Omega=31.2 \mathrm{rad} / \mathrm{s}$. During this time, an image was captured every $5 \mathrm{~min}$, and the orbital radius was measured. The radius obtained as a function of time is shown in Fig. 4.

Because the $\omega_{0}(r)$ relation is very well approximated by a linear function over the range of orbital radii occupied by the dust grain during stage 2 of the experiment (see thick black line in Fig. 3), from Eq. (4), it follows that the grain radius evolution curve $R(t)$ has the same shape as the measured orbital radius curve $r(t)$. Figure 5 depicts the final result of this study, the time evolution of the dust particle size in the discharge plasma. A linear fit to the experimental data results in a reduction rate of $\mathrm{d}\left(R / R_{0}\right) / \mathrm{d} t=-6.7$ $\times 10^{-4} \mathrm{~min}^{-1}$ with a standard deviation of $1.8 \%$.

To support our initial statement, namely, that physical sputtering is the most probable candidate for the underlying mass reduction process, we refer to one of our earlier publications where we have performed a particle in cell with Monte Carlo collisions (PIC/MCC) simulation of the gas discharge including a single dust grain. ${ }^{19}$ These experimentally verified simulations provide access to accurate discharge parameters for very similar discharge conditions to those in our current experiment $\left(p_{\text {sim }}=15 \mathrm{~Pa}, R_{\text {sim }}=5 \mu \mathrm{m}\right.$, and $\left.P_{\text {sim }}=2 \mathrm{~W}\right)$. The computed discharge parameters at to equilibrium position of

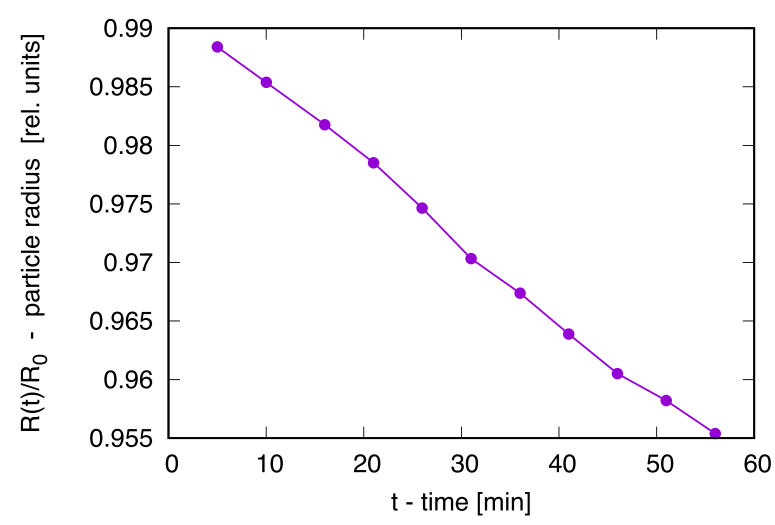

FIG. 5. Evolution of the particle radius relative to its initial size. 
the dust grain include: Debye screening length $\lambda_{\mathrm{D}} \approx 400 \mu \mathrm{m}$, mean ion energy $\left\langle\varepsilon_{i}\right\rangle \approx 1.6 \mathrm{eV}, \mathrm{Ar}^{+}+\mathrm{Ar}$ collision mean free path $\lambda \approx 900 \mu \mathrm{m}$, electron density $n_{e} \approx 6.5 \times 10^{14} \mathrm{~m}^{-3}$, ion density $n_{i} \approx 1.0 \times 10^{15} \mathrm{~m}^{-3}$, and electron temperature $T_{e} \approx 2.0 \mathrm{eV}$.

It is expected that if the dust particle experiences a significant increase in its surface temperature, the evaporation process could become dominant over the physical sputtering. ${ }^{20}$ To estimate the surface temperature, we assume that all ions hitting the dust grain undergo surface recombination and transfer $15.7 \mathrm{eV}$ energy to the surface. The ion flux to the surface is found to be $5.6 \times 10^{12} \mathrm{ion} / \mathrm{s}$ resulting in a heating power of $14.2 \mu \mathrm{W}$. As the distance to the chamber walls is orders of magnitude larger than the grain radius, we approximate the chamber as having spherical symmetry. This allows the temperature rise of the dust surface to be estimated by the general solution of spherical thermal conductivity, $T_{1}=q r_{1}^{2} / k\left(r_{1}^{-1}-r_{2}^{-1}\right)+T_{2}$, where $r_{1}=5 \mu \mathrm{m}$ is the dust grain radius, $r_{2}$ is the distance to the chamber walls $\left(r_{2} \rightarrow \infty\right), q=14.2 \mu \mathrm{W}$ is the heating power, and $k=0.016 \mathrm{~W} /(\mathrm{mK})$ is the thermal conductivity of argon at room temperature. In making this estimation, which includes that only the ions contribute to the heating of the dust surface and only the neutrals contribute to the heat conduction, suggests that the temperature rise of the dust grain cannot be more than $\Delta T=T_{1}-T_{2} \leq 12.8 \mathrm{~K}$. More sophisticated calculations ${ }^{21}$ provide even lower values. This means that no significant temperature rise is expected under these low discharge power conditions and we can safely rule out strong evaporation.

To estimate the uncertainty of our experimental technique we first identify the sources of systematic errors. (i) Stability of the discharge: the voltage probe attached to the powered electrode and the pressure gauge did not pick up any variation over the time of the measurement $(<1 \%$ variation). (ii) Stability of the rotation: the motor has active speed regulation with a nominal accuracy of $\pm 0.2 \%$. (iii) Particle orbit non-circularity: in addition to the circular orbital motion, the particle has peculiar motion resulting in deviations from the perfect circular orbit. The difference between measurements in perpendicular directions is found to be $\leq 5$ pixels in the images. With typical orbital radii above 300 pixels, this effect results in $<2 \%$ uncertainty in the orbital radius reading. (iv) Linear approximation of the $\omega_{0}(r)$ relation: the standard deviation of the fit in Fig. 3 is $<2 \%$. (v) Time and angular velocity measurement: using the computer timer and the timer of the CCD camera it is easy to achieve $<1 \%$ uncertainty here. Altogether we estimate that our primary result, the relative change of the particle radius $\left[R(t) / R_{0}\right]$, has an uncertainty level $<5 \%$, significantly lower than conventional techniques used for the determination of the sputtering yield. ${ }^{22}$

In summary, we conclude that this method is able to capture changes in the order of $0.1 \%$ of the dust particle radius (approximately $5 \mathrm{~nm}$ at a rate of $1 \mathrm{~nm} / \mathrm{min}$ in our demonstrative case using a dust particle with $d=2 R_{0}=8.89 \mu \mathrm{m}$ nominal diameter) and that it is sensitive only to the stability of the gas discharge and the rotation rate. As such, it does not depend on highly uncertain and not directly accessible microscopic discharge or dust particle properties, including optical properties needed for traditional methods. Additionally, micrometer sized dust particles can be made from almost any (conductive and dielectric) solid material. The initial size can be selected simply by sieving or measured with light scattering techniques or by direct imaging. The composition of the discharge gas can also be selected according to the needs of the system of interest, making this method a universal tool for future studies of the sputtering process in gas discharges.

This work has been supported in Hungary by the NKFIH Grant No. K-115805 and the János Bolyai Research Scholarship of the Hungarian Academy of Sciences. The technical assistance of Tibor Szücs (Wigner RCP) and Mike Cook (CASPER) is gratefully acknowledged.

${ }^{1}$ Plasma-Surface Interactions and Processing of Materials, NATO ASI Series E: Applied Sciences Vol. 176, edited by O. Auciello, A. GrasMartí, J. A. Valles-Abarca, and D. L. Flamm (Kluwer Academic Publisher, 1990).

${ }^{2}$ H. S. W. Massey, E. H. S. Burhop, and H. B. Gilbody, Electronic and Ionic Impact Phenomena Vol. III: Slow Collisions of Heavy Particles (Clarendon Press, Oxford, 1971).

${ }^{3}$ W. Jacob, Thin Solid Films 326, 1-42 (1998).

${ }^{4} \mathrm{M}$. Bonitz, "Towards a unified treatment of the plasma-solid system. The Kiel research project 'The Plasma Interface'," in Conference Abstract, Quo Vadis - Complex Plasmas Summers School and Conference, Hamburg, Germany, 1-4 August 2016.

${ }^{5} \mathrm{R}$. Behrisch, Sputtering by Particle Bombardment I-III (Springer Verlag, Berlin, 1981/1991).

${ }^{6}$ P. Hartmann, A. Zs. Kovács, J. C. Reyes, L. S. Matthews, and T. W. Hyde, Plasma Sources Sci. Technol. 23, 045008 (2014).

${ }^{7}$ J. Carstensen, F. Greiner, and A. Piel, Phys. Plasmas 17, 083703 (2010).

${ }^{8}$ M. Bonitz, C. Henning, and D. Block, Rep. Prog. Phys. 73, 066501 (2010).

${ }^{9}$ G. Gebauer and J. Winter, New J. Phys. 5, 38 (2003).

${ }^{10}$ R. L. Heinisch, F. X. Bronold, and H. Fehske, Phys. Rev. Lett. 109, 243903 (2012).

${ }^{11}$ W. W. Stoffels, E. Stoffels, G. H. P. M. Swinkels, M. Boufnichel, and G. M. W. Kroesen, Phys. Rev. E 59, 2302 (1999).

${ }^{12}$ C. Killer, M. Mulsow, and A. Melzer, Plasma Sources Sci. Technol. 24, 025029 (2015).

${ }^{13}$ C. Killer, F. Greiner, S. Groth, B. Tadsen, and A. Melzer, Plasma Sources Sci. Technol. 25, 055004 (2016).

${ }^{14}$ J. Carstensen, H. Jung, F. Greiner, and A. Piel, Phys. Plasmas 18, 033701 (2011).

${ }^{15}$ H. Jung, F. Greiner, O. H. Asnaz, J. Carstensen, and A. Piel, J. Plasma Phys. 82, 615820301 (2016).

${ }^{16}$ J. Pavlu, A. Velyhan, I. Richterova, Z. Nemecek, J. Safrankova, I. Cermak, and P. Zilavy, IEEE Trans. Plasma Sci. 32, 704 (2004).

${ }^{17}$ X. Z. Tang and G. L. Delzanno, Phys. Plasmas 21, 123708 (2014).

${ }^{18}$ S. Hamaguchi and R. T. Farouki, Phys. Rev. E 49, 4430 (1994).

${ }^{19}$ N. Kh. Bastykova, A. Zs. Kovács, I. Korolov, S. K. Kodanova, T. S. Ramazanov, P. Hartmann, and Z. Donkó, Contrib. Plasma Phys. 55, 671-676 (2015).

${ }^{20}$ M. A. Ermolenko, E. S. Dzlieva, V. Yu. Karasev, S. I. Pavlov, V. A. Polishchuk, and A. P. Gorbenko, Tech. Phys. Lett. 41, 1199-1201 (2015).

${ }^{21}$ S. A. Khrapak and G. E. Morfill, Phys. Plasmas 13, 104506 (2006).

${ }^{22}$ Sputtering by Particle Bombardment: Experiments and Computer Calculations from Threshold to MeV Energies, edited by R. Behrisch and W. Eckstein (Springer, 2007). 